\title{
Molecular Neurobiology \\ Up-regulation of Oligodendrocyte Lineage Markers in the Cerebellum of Autistic Patients: Evidence from Network Analysis of Gene Expression \\ --Manuscript Draft--
}

\begin{tabular}{|c|c|}
\hline Manuscript Number: & MOLN-D-15-00235R1 \\
\hline Article Type: & Original Article \\
\hline Keywords: & $\begin{array}{l}\text { ASD; psychiatric disorders; white matter; glia; oligodendrocytes; connectivity; } \\
\text { development. }\end{array}$ \\
\hline Corresponding Author: & $\begin{array}{l}\text { Fares Zeidán-Chuliá, Ph.D. } \\
\text { BRAZIL }\end{array}$ \\
\hline First Author: & Fares Zeidán-Chuliá, Ph.D. \\
\hline \multirow[t]{7}{*}{ Order of Authors: } & Fares Zeidán-Chuliá, Ph.D. \\
\hline & Ben-Hur Neves de Oliveira \\
\hline & Manuel F. Casanova \\
\hline & Emily L. Casanova \\
\hline & Mami Noda \\
\hline & Alla B. Salmina \\
\hline & Alexei Verkhratsky \\
\hline Abstract: & $\begin{array}{l}\text { Autism is a neurodevelopmental disorder manifested by impaired social interaction, } \\
\text { deficits in communication skills, restricted interests, and repetitive behavior. In } \\
\text { neurodevelopmental, neurodegenerative, and psychiatric disorders, glial cells undergo } \\
\text { morphological, biochemical, and functional rearrangements, which are critical for } \\
\text { neuronal development, neurotransmission, and synaptic connectivity. Cerebellar } \\
\text { function is not limited to motor coordination but also contributes to cognition and may } \\
\text { be affected in autism. Oligodendrocytes and specifically oligodendroglial precursors } \\
\text { are highly susceptible to oxidative stress and excitotoxic insult. In the present study, we } \\
\text { searched for evidence for developmental oligodendropathy in the context of autism by } \\
\text { performing a network analysis of gene expression of cerebellar tissue. We created an } \\
\text { in silico network model (OLIGO) showing the landscape of interactions between } \\
\text { oligodendrocyte markers and demonstrated that more than } 50 \% \text { (16 out of } 30 \text { ) of the } \\
\text { genes within this model displayed significant changes of expression (corrected p-value } \\
<0.05 \text { ) in the cerebellum of autistic patients. In particular, we found up-regulation of } \\
\text { OLIG2, MBP, OLIG1, and MAG specific oligodendrocyte markers. We postulate that } \\
\text { aberrant expression of oligodendrocyte-specific genes, potentially related to changes } \\
\text { in oligodendrogenesis, may contribute to abnormal cerebellar development, impaired } \\
\text { myelination, and anomalous synaptic connectivity in autism spectrum disorders (ASD). }\end{array}$ \\
\hline
\end{tabular}




\section{Prof. Nicolas G. Bazan}

Louisiana State University Health Sciences Center

New Orleans, LA, USA

Editor-in-chief (Molecular Neurobiology)

Re: MOLN-D-15-00235

Dear Prof. Bazan,

We shall be very much obliged if you may consider the revised version of our research article entitled "Up-regulation of oligodendrocyte lineage markers in the cerebellum of autistic patients: Evidence from network analysis of gene expression" by Fares Zeidán-Chuliá, Ben-Hur Neves de Oliveira, Manuel F. Casanova, Emily L. Casanova, Mami Noda, Alla B. Salmina, and Alexei Verkhratsky for publication in Molecular Neurobiology. Please, find below point-by-point responses and a list of improvements based on the referee's suggestions. We are grateful to the reviewer's positive comments regarding our manuscript.

With best regards.

Yours sincerely,

Fares Zeidán-Chuliá (on behalf of all authors)

E-mail: fzchulia.biomed@gmail.com 


\section{Re: MOLN-D-15-00235}

\section{RESPONSE TO REVIEWERS}

\section{COMMENTS FOR THE AUTHOR:}

Reviewer \#2: The paper entitled $» U p$-regulation of oligodendrocyte lineage markers in the cerebellum of autistic patients: Evidence from network analysis of gene expression" by Fares Zeidán-Chuliá, Ben-Hur Neves de Oliveira, Manuel F. Casanova, Emily L. Casanova, Mami Noda, Alla B. Salmina, and Alexei Verkhratsky, addresses the neuropathological mechanisms of autism from different perspectives including oxidative stress, genetics, neuropathology, and neuron-glia biology. The manuscript presents evidence for altered expression of genes potentially related to changes in oligodendrogenesis, which may contribute to abnormal cerebellar development, impaired myelination, and aberrant synaptic connectivity observed by others in autistic patients. The approach is based on using experimental data and database computational analyses to develop an in silico model, under a high confidence score and a false discovery rate filter for the network-focused gene expression analysis. What is unusual about this paper is that it dwels on the hypothesis that oligodendroglia are playing a role in autism. The results of this in silico study indentified that 17 out of 30 studied genes within this model displayed significant changes of expression (corrected p-value $<0.05$ ) in the cerebellum of autistic patients. These include OLIG2, MBP, OLIG1, and MAG, all oligodendrocyte markers. Based on these findings, the authors hypothesize that up-regulation of genes related to oligodendrocyte lineage may represent an adaptive response to stress-induced cellular loss, and possibly reflects aberrant oligodendrogenesis and/or oligodendrocyte maturation in the cerebellum of autistic patients. This hypothesis is useful for further experimentla studies. Five laboratories collaborate on this rather crisp paper, which reads well. There is only one very minor suggestions. One connector gene is crc, a viral gene. How is this gene related to the pathogenesis of autism? A short discussion would be appropriate to address the possibility that autism may in part bi associated also to viral infection?

Our reply: we thank the referee for the positive comments about our study. We assume the referee's question relates to $v$-crk gene (sarcoma virus CD10 oncogene homolog; and not "crc") and how this viral gene may be related to the pathogenesis of autism. This is indeed an excellent point. Crk proteins link upstream tyrosine kinase and integrin-dependent signals to downstream effectors, acting as adaptors in diverse signaling pathways and cellular processes, and its role is well-described in cancer cells (Bell and Park, 2012). One proposed etiology for autism is viral infection very early in development. A number of studies over the years have presented evidence both for and against the association of autism with various viral infections (Libbey et al., 2005). 
Bell ES, Park M (2012) Models of crk adaptor proteins in cancer. Genes Cancer 3(56):341-352.

Libbey JE, Sweeten TL, MacMahon WM, Fujinami RS (2005) Autistic disorder and viral infections. J Neurovirol 11(1):1-10.

Of note, Src and v-crk activities (Bell and Park, 2012) are connected through the crkassociated substrate (CAS). Therefore, one could speculate that migration and adhesion of oligodendrocytes or their precursors might be somehow affected. It is a very much intriguing and interesting observation by the referee, so we have added it as a part of the discussion section (Page 8, first paragraph) together with two additional references (36 and 37). Moreover, we will certainly approach it in the next two upcoming studies we are preparing regarding the potential role of oligodendrocytes in the developmental pathogenesis of autism.

Reviewer \#3: The paper presents evidence for altered expression of oligodendrocyte genes associated with autistm spectrum disorders (ASD). The study mines microarray data from the Geo DataSets database that were originally contributed by Ginsberg and colleagues (GSE38322), and applies an in silico network model of interactions of oligodendrocyte markers ("OLIGO"), based on a systematic review of pubmed. It is of course essential that this Oligo network is made fully available as part of this paper, although could not see that this was the case. Presently, the results present a summary only and the full material should be provided as supplementary material for the use of readers of the paper. For example, a number of key genes appear to be absent from the analysis, e.g. Sox10, which is sufficient and necessary for oligodendrocyte specification, Gpr17, which regulates the timing of myelination, and Nkx2.2 which is expressed by OPCs and throughout the oligodendrocyte lineage. The key findings are that a number of essential myelin genes are increased in ASD (although other key genes that are essential for myelination are unaltered, such as PLP1, CNP, GalC and MOG). The increase in Cspg4 and Pdgfra are also interesting, because these imply increases in OPCs, but other OPC markers should also be analysed, such as Nkx2.2. Based on the findings, the authors propose a model in which up-regulation of oligodendroglial genes may represent an adaptive response to stress-induced cellular loss, and possibly reflects aberrant oligodendrogenesis and/or oligodendrocyte maturation in the cerebellum of autistic patients. This conclusion would be strengthened considerably by qPCR to validate these findings and immunohistochemical analysis to determine whether the microarray data reflects changes in cells and protein.

Our reply: we thank the referee for the comments. Please, kindly note that we did not have provided a resume of any kind in the present short report. As a matter of fact, every detail about how the network was constructed input options for the STRING database, confidence score, and all gene IDs are provided in the main manuscript text and table 1 to allow the readers to re-create our model. The referee is completely right about the qPCRs and immunohistochemical staining. We are currently planning to perform these and other 
experiments in an in vivo model of autism to dig further in the molecular basis of the present findings as part of another post-graduate student's thesis from our group.

Reviewer \#4: In this paper, authors test an interesting and novel hypothesis: the presence of myelin dysfunction in autism. Very few studies have so far focused on myelin as a specific target for developmental and connectivity diseases associated to marked neuropsychiatric symptoms such as autism. In this respect, this is indeed one of the first pioneer studies in the area. However, the problem with this paper is that, despite being an Original Research report, there is no experimental research in it, apart from a descriptive in silico analysis. Specifically, authors generated an in silico network model composed by 30 genes connecting some highly specific oligodendroglial genes with 17 additional "connectors". They then utilized this model to analyse microarray data from cerebellar samples of autistic patients compared to healthy subjects originally contributed by Ginsberg and colleagues (REF 21). Analysis revealed differential expression of the identified targets, with an upregulation of myelin genes. These data are of course highly interesting but, besides being only descriptive, in my opinion they should have been used as a starting point for a more in depth study on in vitro or in vivo models of autism, to understand how specific these changes are, and unveil the functional relationships between the different identified genes.

Our reply: we sincerely think the referee for the positive comments about our study. We completely agree. The present data and hypothesis is rather new and unusual within the ASD context. Deciphering of new molecular targets for therapy and development of novel transgenic models of ASD would be a beneficial outcome from these findings and it is something we are planning to do as part of another post-graduate student's thesis from our group. 


\section{Up-regulation of Oligodendrocyte Lineage Markers in the Cerebellum of Autistic Patients: Evidence from Network Analysis of Gene Expression}

Fares Zeidán-Chuliá ${ }^{*}$, Ben-Hur Neves de Oliveira ${ }^{1}$, Manuel F. Casanova ${ }^{2}$, Emily L. Casanova $^{2}$, Mami Noda ${ }^{3}$, Alla B. Salmina ${ }^{4}$, Alexei Verkhratsky $5,6,7$

${ }^{1}$ Departamento de Bioquímica, Instituto de Ciências Básicas da Saúde, Universidade Federal do Rio Grande do Sul (UFRGS), Porto Alegre, Brazil

${ }^{2}$ Department of Psychiatry \& Behavioral Sciences, University of Louisville, Louisville, KY, USA

${ }^{3}$ Laboratory of Pathophysiology, Graduate School of Pharmaceutical Sciences, Kyushu University, Fukuoka, Japan

${ }^{4}$ Department of Biochemistry, Medical, Pharmaceutical \& Toxicological Chemistry, Krasnoyarsk State Medical University, Krasnoyarsk, Russia

${ }^{5}$ Faculty of Life Sciences, The University of Manchester, Manchester, UK

${ }^{6}$ IKERBASQUE, Basque Foundation for Science, Bilbao, Spain and Department of Neurosciences, University of the Basque Country UPV/EHU, Leioa, Spain

${ }^{7}$ University of Nizhny Novgorod, Nizhny Novgorod, Russia

* Corresponding Author: Dr. Fares Zeidán Chuliá; Departamento de Bioquímica, Instituto de Ciências Básicas da Saúde, Rua Ramiro Barcelos, 2600-anexo-Bairro Santana, 90035-003 Porto Alegre, RS, Brasil. Tel.: +55 513308 5577; fax: +55 513308 5535. Email: fzchulia.biomed@gmail.com

Short Title: Oligodendrocyte Lineage Markers, Cerebellum, and Autism.

Keywords: ASD; psychiatric disorders; white matter; glia; oligodendrocytes; connectivity; development.

Abbreviations: APP, amyloid protein precursor; ASD, autism spectrum disorders; CNS, central nervous system; FDR, false discovery rate; OL, oligodendrocyte; OPCs, oligodendroglial precursors. 


\section{ABSTRACT}

Autism is a neurodevelopmental disorder manifested by impaired social interaction, deficits in communication skills, restricted interests, and repetitive behavior. In neurodevelopmental, neurodegenerative, and psychiatric disorders, glial cells undergo morphological, biochemical, and functional rearrangements, which are critical for neuronal development, neurotransmission, and synaptic connectivity. Cerebellar function is not limited to motor coordination but also contributes to cognition and may be affected in autism. Oligodendrocytes and specifically oligodendroglial precursors are highly susceptible to oxidative stress and excitotoxic insult. In the present study, we searched for evidence for developmental oligodendropathy in the context of autism by performing a network analysis of gene expression of cerebellar tissue. We created an in silico network model (OLIGO) showing the landscape of interactions between oligodendrocyte markers and demonstrated that more than $50 \%$ (16 out of 30) of the genes within this model displayed significant changes of expression (corrected $p$-value $<0.05)$ in the cerebellum of autistic patients. In particular, we found up-regulation of $O L I G 2, M B P, O L I G 1$, and $M A G$ specific oligodendrocyte markers. We postulate that aberrant expression of oligodendrocyte-specific genes, potentially related to changes in oligodendrogenesis, may contribute to abnormal cerebellar development, impaired myelination, and anomalous synaptic connectivity in autism spectrum disorders (ASD). 


\section{Introduction}

Despite an increasing prevalence of autistic spectrum disorders (for example, 1 in every 50 children in the United States) [1], there is, hitherto, no definitive consensus about the etiology of autism, although the role for both environmental and genetic factors is generally accepted [2,3]. Pathogenic factors of autism include: (i) increased oxidative stress [4], (ii) glial activation [5], (iii) abnormal amyloid protein precursor (APP) processing [6], (iv) minicolumn pathology [7], (v) and deregulation of neurogenesis, neuronal migration, and neuronal maturation [8]. The variety of pathophysiological mechanisms, their succession and timing, and the individual susceptibility of the particular individual may all combine to account for the heterogeneity of clinical phenotypes [8,9]. The abnormal enlargement of the brain observed in children with autism spectrum disorders (ASD) [10] has been linked to changes in both grey and white matter [11], with the latter being, arguably, predominantly affected [12]. White matter overgrowth has been linked to excessive axonogenesis, glial proliferation and/or excessive myelination [13]. There is emerging agreement about the role of cerebellum in the etiopathogenesis of autism. Points of consensus include the abnormal neurotransmission, oxidative stress, cerebellar motor and cognitive deficits, as well as neuroinflammation, or abnormal cerebellar anatomy in autistic patients [14]. Differences in the total volume of the cerebellum and the cerebellar hemispheres (that in some studies were found to be significantly larger with and without correcting for total brain volume in non-mentally retarded autistic adolescents and adults) have been already been identified [15]. Abnormal cerebellar circuitry and output may instigate anomalous development of neurobehavioral systems linked to the cerebellum represented, for instance, by motor control systems, limbic and language systems, hypothalamus, thalamus, parietal and frontal cortex. Similarly, aberrant cerebellar 
output may affect serotonergic, noradrenergic, and dopaminergic transmission [16]. In this study, we sought evidence for altered oligodendrogenesis that may affect cerebellar development in ASD. Based on our findings, we hypothesize that up-regulation of genes related to oligodendrocyte (OL) lineage may represent an adaptive response to stress-induced cellular loss, and possibly reflects aberrant oligodendrogenesis and/or oligodendrocyte maturation in the cerebellum of autistic patients.

\section{Material and Methods}

Development of the Gene/Protein Network Model, Data Acquisition and Processing

We created an in silico network model of interactions of oligodendrocyte markers (“OLIGO”). We performed a systematic review of both original research articles and reviews by searching for literature associated with this topic in the PubMed database (http://www.ncbi.nlm.nih.gov/pubmed/). These studies were obtained by combining the terms “oligodendrocyte”, “marker”, "biomarker”, and "expression”. Thereafter, we searched for the possible landscape of interactions between these genes/proteins by using the on line search tool for the retrieval of interacting genes STRING 9.1 (http://string-db.org/) [17]; with exclusively “Experiments"' and "Databases" as input options for active prediction methods and a high confidence score of 0.600 (0.400 is considered as medium confidence). The links (interaction strength) between two different genes/proteins (nodes) were saved in data files to be handled in the Medusa software [18]. The in silico model "OLIGO" is composed of (i) genes corresponding to proteins typically expressed in oligodendrocytes (some of those being specific oligodendrocyte markers, such as $O L I G 1, O L I G 2, M B P$, and $M A G)[19,20]$ and (ii) 
additional genes/proteins (named as "connectors"), provided by the search tool for interconnecting these markers within the in silico network.

Determination of Differentially Expressed Genes, Statistics, and Relative Gene Expression Network Visualization

Microarray data (raw data) were obtained from the Geo DataSets database (http://www.ncbi.nlm.nih.gov/geo/). These data were originally contributed by Ginsberg and colleagues (GSE38322) [21]. The database is publicly available, and contains data obtained from cerebellar hemispheric cortex of patients with autism and control individuals. Experimental assays are explained in detail in the original publication; which also provides information on the selection criteria of patients (inclusion and exclusion) and diagnostic profile [21]. For determining the differential expression of members from the "OLIGO" network, expression data were filtered from probes with $<0.05$ signal detection $p$-values and normalized by using the lumi package from $\mathrm{R}$ [22] and robust spline normalization. Thereafter, differential gene expression was elucidated by analyzing, with the limma package from $\mathrm{R}$ and false discovery rate (FDR) [23], normalized data from diseased cerebellar samples vs. healthy controls. One key statistical shift is always the move from the well known $P$-value to false discovery rate (FDR), in order to obtain a corrected $P$-value. FDR is a general method for accurate estimation of false discovery rates [23]. Only genes with corrected $p$-values $<0.05$ were considered statistically significant. Gene expression visualization over the "OLIGO" network was performed by utilizing the ViaComplex software (http://lief.if.ufrgs.br/pub/biosoftwares/viacomplex/) [3,6]. 


\section{Results and Discussion}

We have constructed the in silico model that resulted in a network of interactions composed by 30 genes/proteins (13 OL selected genes/proteins and 17 additional genes/proteins or "connectors") based on "Experiments" and "Databases" and a confidence score of 0.600 (Fig. 1A). This model was then subjected to further analysis by the ViaComplex software, which plots the relative expression derived from each cerebellar sample from patients with autism over the expression of healthy ones ( $Z$-axis) in the "OLIGO" network in 2D and 3D views (Fig. 1B and C). Differential expression analysis of "OLIGO" network revealed transcriptional changes in the cerebellar hemispheric cortex of autistic individuals in 16 out of 30 genes (Table 1). Among them, proteins being specific for OLs such as $O L I G 2, M B P, O L I G 1$, and $M A G$ together with other differentially expressed genes (i.e., CDC42, PTK2, PRMT1, PDGFRA, CSPG4, $F Y N, T J P 1, Q K I, G J C 1, G R B 2, G L A$, and EP300) in these tissue samples (corrected $p$ values <0.05) (Table 1). The observed changes may account for the aberrant oligodendrogenesis and myelination with consequent alterations in brain connectivity, characteristic to ASD.

While nearly all neurons are in place at birth, myelination occurs mainly postnatally and white matter volume increases throughout childhood, adolescence, and adulthood [24]. When OLs (responsible for myelination) are ablated, cerebellar development is impaired $[25,26]$. OLs and especially oligodendroglial precursors (OPCs) are highly susceptible to oxidative stress-induced cell death. This is linked to high levels of iron required for myelin synthesis, low levels of reduced-glutathione, poor ability to scavenge peroxides and relatively high expression of $\mathrm{Ca}^{2+}$ permeable glutamate receptors $[27,28]$. Exposure to environmental stress that reduces numbers of 
neurons and OLs may lead to defects in cerebellar synaptogenesis and postnatal myelinization. Since OPCs seem to be significantly more sensitive to the toxic effects of glutathione depletion when compared to OLs [29], the maturation-dependent susceptibility to oxidative stress could be a major factor in the pathogenesis of some neurodevelopmental disorders, including ASD [29,30].

Clinical symptoms of autism are often preceded by two phases of aberrant growth of the brain: a reduced head size at birth which is followed by a sudden and excessive growth of the brain between 1 to 2 months and 6 to 14 months of age [11]. At 2 to 4 years, the most significant growth can be observed in the frontal lobe, limbic, and cerebellar structures $[11,15]$. Variability in cerebellar and cerebral size correlates with functional deficits in children with ASD (aged between 2-5 years) [31]. Brain overgrowth could be an indicator of increased neurogenesis and/or defects in developmental apoptosis [32]. Brain enlargement shows greater increase in white matter in both cerebrum and cerebellum in young children with ASD [12,31,33]. Since white matter is composed of glial cells and myelinated axons, one could speculate that the predominant overgrowth of white matter could be a sign of increased myelination. Recent evidence point towards this direction with reports showing higher myelination of the corpus callosum in both the left and right medial frontal cortex in ASD [34,35]. Cerebellar white matter hyperplasia is also present in early life of patients with ASD [11,31]. Our results may further corroborate an increased myelination in the cerebellar cortex of these patients wherein the up-regulation $Q K I, M B P$, and $M A G$ genes correspond to myelinating OL markers (Table 1). In spite of the present findings, the absence of experiments confirming the up-regulation of these genes (at the protein level) in the cerebellar hemispheric cortex of ASD patients remains a limitation that requires further investigation and the use of additional cohorts of autistic patients. 
Finally, one proposed theory for the etiology for autism is viral infection very early in development. A number of studies over the years have presented evidence both for and against the association of autism with viral infections [36]. Incidentally, one member (connector) of our network model is CRK (v-crk sarcoma virus CT10 oncogene homolog) (Table 1). Crk proteins link upstream tyrosine kinase and integrin-dependent signals to downstream effectors, acting as adaptors in diverse signaling pathways and cellular processes, and its role is well-described in cancer cells [37]. Of note, Src and vcrk activities [37] are connected through the crk-associated substrate (CAS). Therefore, one could speculate whether migration and adhesion of oligodendrocytes or their precursors might be somehow affected and may worth further investigation.

\section{Conclusions}

We here hypothesize (Fig. 2) that (i) the up-regulation in expression of oligodendrocyte lineage marker genes (and possibly oligodendrogenesis), (ii) increased local short-range connectivity, and (iii) weaker long-range connectivity along the cerebello-thalamocortical circuit would be a model that may explain many of the behavioral and cognitive deficits present in ASD. The latter findings could represent an adaptive response to stress-induced cell death and would support the local hyperconnectivity-associated excitatory synaptogenesis in the cerebellum [38].

Acknowledgements. FZC holds a PNPD postdoctoral position (Programa de Pós Graduação em Ciências Biológicas: Bioquímica, Universidade Federal do Rio Grande do Sul) funded by CAPES. AV was supported in part by the grant (agreement from August 272013 No. 02.B.49.21.0003) between The Ministry of Education and Science 
of the Russian Federation and Lobachevsky State University of Nizhny Novgorod and by the grant of the Russian Scientific Foundation No.14-15-00633.

Conflict of Interest. The authors declare that they have no conflict of interest. 


\section{References}

1. Blumberg SJ, Bramlett MD, Kogan MD, Schieve LA, Jones JR (2013) Changes in prevalence of parent-reported autism spectrum disorder in school-aged U.S. children: 2007 to 2011-2012. National Health Statistics Reports 65:1-12.

2. Zeidán-Chuliá F, Gursoy UK, Könönen E, Gottfried C (2011) A dental look at the autistic patient through orofacial pain. Acta Odontol Scand 69(4):193-200.

3. Zeidán-Chuliá F, Rybarczyk-Filho JL, Salmina AB, de Oliveira BH, Noda M, Moreira JC (2013) Exploring the Multifactorial Nature of Autism Through Computational Systems Biology: Calcium and the Rho GTPase RAC1 under the spotlight. Neuromolecular Med 15(2):364-383.

4. Chauhan A, Chauhan V (2006) Oxidative stress in autism. Pathophysiology 13(3):171-181.

5. Zeidán-Chuliá F, Salmina AB, Malinovskaya NA, Noda M, Verkhratsky A, Moreira JC (2014) The glial perspective of autism spectrum disorders. Neurosci Biobehav Rev 38:160-172.

6. Zeidán-Chuliá F, de Oliveira BH, Salmina AB, Casanova MF, Gelain DP, Noda M, Verkhratsky A, Moreira JC (2014) Altered expression of Alzheimer's disease-related genes in the cerebellum of autistic patients: a model for disrupted brain connectome and therapy. Cell Death Dis 5:e1250.

7. Casanova MF, Buxhoeveden DP, Brown C (2002) Clinical and macroscopic correlates of minicolumnar pathology in autism. J Child Neurol 17(9):692-695.

8. Wegiel J, Kuchna I, Nowicki K, Imaki H, Wegiel J, Marchi E, Ma SY, Chauhan A, et al (2010) The neuropathology of autism: defects of neurogenesis and neuronal migration, and dysplastic changes. Acta Neuropathol 119(6):755-770. 
9. Jeste SS, Geschwind DH (2014) Disentangling the heterogeneity of autism spectrum disorder through genetic findings. Nat Rev Neurol 10(2):74-81.

10. Shen MD, Nordahl CW, Young GS, Wootton-Gorges SL, Lee A, Liston SE, Harrington KR, Ozonoff S, et al (2013) Early brain enlargement and elevated extra-axial fluid in infants who develop autism spectrum disorder. Brain 136(Pt 9):2825-2835.

11. Courchesne E, Karns CM, Davis HR, Ziccardi R, Carper RA, Tique ZD, Chisum HJ, Moses P, et al (2001) Unusual brain growth patterns in early life in patients with autistic disorder: an MRI study. Neurology 57(2):245-254.

12. Amaral DG, Schumann CM, Nordahl CW (2008) Neuroanatomy of autism. Trends Neurosci 31(3):137-145.

13. Courchesne E, Pierce K (2005) Why the frontal cortex in autism might be talking only to itself: Local overconnectivity but long-distance disconnection. Curr Opin Neurobiol 15(2):225-230.

14. Fatemi SH, Aldinger KA, Ashwood P, Bauman ML, Blaha CD, Blatt GJ, Chauhan A, Chauhan V, et al (2012) Consensus paper: pathological role of the cerebellum in autism. Cerebellum 11(3):777-807.

15. Hardan AY, Minshew NJ, Harenski K, Keshavan MS (2001) Posterior fossa magnetic resonance imaging in autism. J Am Acad Child Adolesc Psychiatry 40(6):666-672.

16. Courchesne E (1997) Brainstem, cerebellar and limbic neuroanatomical abnormalities in autism. Curr Opin Neurobiol 7(2):269-278.

17. Szklarczyk D, Franceschini A, Kuhn M, Simonovic M, Roth A, Minguez P, Doerks T, Stark M, et al (2011) The STRING database in 2011: Functional 
interaction networks of proteins, globally integrated and scored. Nucleic Acids Res 39(Database issue):D561-D568.

18. Hooper SD, Bork P (2005) Medusa: a simple tool for interaction graph analysis. Bioinformatics 21(24):4432-4433.

19. Baumann N, Pham-Dinh D (2001) Biology of oligodendrocyte and myelin in the mammalian central nervous system. Physiol Rev 81(2):871-927.

20. Deng W, Poretz RD (2003) Oligodendroglia in developmental neurotoxicity. Neurotoxicology 24(2):161-178.

21. Ginsberg MR, Rubin RA, Falcone T, Ting AH, Natowicz MR (2012) Brain transcriptional and epigenetic associations with autism. PLoS One 7(9):e44736.

22. Gentleman RC, Carey VJ, Bates DM, Bolstad B, Dettling M, Dudoit S, Ellis B, Gautier L, et al (2004) Bioconductor: Open software development for computational biology and bioinformatics. Genome Biol 5(10):R80.

23. Pawitan Y, Michiels S, Koscielny S, Gusnanto A, Ploner A (2005) False discovery rate, sensitivity and sample size for microarray studies. Bioinformatics 21(13):3017-3024.

24. Walhovd KB, Johansen-Berg H, Karadottir RT (2014) Unraveling the secrets of white matter--Bridging the gap between cellular, animal and human imaging studies. Neuroscience 276: 2-13.

25. Mathis C, Collin L, Borrelli E (2003) Oligodendrocyte ablation impairs cerebellum development. Development 130(19):4709-4718.

26. El Waly B, Macchi M, Cayre M, Durbec P (2014) Oligodendrogenesis in the normal and pathological central nervous system. Front Neurosci 8:145.

27. Verkhratsky A, Kirchhoff F (2007) NMDA Receptors in glia. Neuroscientist 13(1):28-37. 
28. Thorburne SK, Juurlink BH (1996) Low glutathione and high iron govern the susceptibility of oligodendroglial precursors to oxidative stress. J Neurochem 67(3):1014-1022.

29. Back SA, Gan X, Li Y, Rosenberg PA, Volpe JJ (1998) Maturation-dependent vulnerability of oligodendrocytes to oxidative stress-induced death caused by glutathione depletion. J Neurosci 18(16):6241-6253.

30. Butts BD, Houde C, Mehmet H (2008) Maturation-dependent sensitivity of oligodendrocyte lineage cells to apoptosis: implications for normal development and disease. Cell Death Differ 15(7):1178-1186.

31. Akshoomoff N, Lord C, Lincoln AJ, Courchesne RY, Carper RA, Townsend J, Courchesne E (2004) Outcome classification of preschool children with autism spectrum disorders using MRI brain measures. J Am Acad Child Adolesc Psychiatry 43(3):349-357.

32. Raff MC, Barres BA, Burne JF, Coles HS, Ishizaki Y, Jacobson MD (1993) Programmed cell death and the control of cell survival: lessons from the nervous system. Science 262(5134):695-700.

33. Hendry J, DeVito T, Gelman N, Densmore M, Rajakumar N, Pavlosky W, Williamson PC, Thompson PM, et al (2006) White matter abnormalities in autism detected through transverse relaxation time imaging. Neuroimage 29(4): 1049-1057.

34. Carmody DP, Lewis M (2010) Regional white matter development in children with autism spectrum disorders. Dev Psychobiol 52(8):755-763.

35. Gozzi M, Nielson DM, Lenroot RK, Ostuni JL, Luckenbaugh DA, Thurm AE, Giedd JN, Swedo SE (2012) A magnetization transfer imaging study of corpus 
callosum myelination in young children with autism. Biol Psychiatry 72(3):215220.

36. Libbey JE, Sweeten TL, MacMahon WM, Fujinami RS (2005) Autistic disorder and viral infections. J Neurovirol 11(1):1-10.

37. Bell ES, Park M (2012) Models of crk adaptor proteins in cancer. Genes Cancer $3(5-6): 341-352$.

38. Gibson EM, Purger D, Mount CW, Goldstein AK, Lin GL, Wood LS, Inema I, Miller SE, et al (2014) Neuronal activity promotes oligodendrogenesis and adaptive myelination in the mammalian brain. Science 344(6183):1252304. 


\section{Figure legends}

Fig. 1 "OLIGO" network analysis. General landscape of interactions between genes/proteins expressed in oligodendrocytes. (A) The in silico network model was developed by using the STRING 9.1 database resource search tool, exclusively using "Experiments" and "Databases" as input options for active prediction methods and a confidence score of 0.600 . (B) Relative gene expression over the in silico model "OLIGO" (cerebellar samples of autistic patients vs. healthy controls) represented by using the ViaComplex software, which is an open-source application that builds landscape maps of gene expression networks.

Fig. 2 Possible role of up-regulated cerebellar OL lineage markers in the pathogenesis of autism. In the present model, we suggest that increased OLs susceptibility to environmental stress, together with the genetic contribution associated to autism, may lead to defects in cerebellar synaptogenesis and postnatal myelination. The adaptive response of the CNS to stress-induced cell death would include increased neurogenesis with promotion of oligodendrogenic transcriptional program in an attempt to compensate the decreased number in cells and myelin deposition. Such response may possibly explain the increased cerebellar volume (consistent with the increase in regional and total brain size) and white matter hyperplasia described in these patients. The cerebellum projects, via the thalamus, to the prefrontal cortex which in turn innervates cerebellar areas from where prefrontal cortical projections are originated. We postulate that (i) up-regulation in expression of oligodendrocyte lineage marker genes (and possibly oligodendrogenesis) (ii) increased local short-range connectivity and (iii) weaker long-range connectivity along the cerebello-thalamo-cortical circuit may explain many of the behavioral and cognitive hallmarks of ASD. The up-regulation in 
expression of OL lineage marker genes (and possibly oligodendrogenesis) could be explained as an adaptive response to stress-induced cellular loss and would support the local hyperconnectivity-associated excitatory synaptogenesis. 
Table 1 Differential gene expression profile of the "OLIGO" network members in autism (cerebellum). Values are considered significant when corrected $p$-values $<0.05$ (FDR).

\begin{tabular}{|c|c|c|c|c|c|}
\hline $\begin{array}{c}\text { Gene } \\
\text { symbol }\end{array}$ & $\begin{array}{c}\text { Alias } \\
\text { and/or } \\
\text { description }\end{array}$ & $\begin{array}{l}\text { Ensembl ID } \\
\text { (ENSP) }\end{array}$ & $\begin{array}{c}\text { Network } \\
\text { node status }\end{array}$ & $\begin{array}{c}\text { Corrected } \\
p \text {-value } \\
\text { (FDR) }\end{array}$ & $\begin{array}{c}\text { Differential } \\
\text { expression }\end{array}$ \\
\hline$C D C 42$ & Cell division cycle 42 (GTP binding protein, $25 \mathrm{kDa}$ ) & ENSP00000314458 & Connector & 0.002000135 & DOWN \\
\hline PRMT1 & Protein arginine methyltransferase 1 & ENSP00000246789 & Connector & 0.002437104 & DOWN \\
\hline PDGFRA & Platelet-derived growth factor receptor, $\alpha$ polypeptide & ENSP00000257290 & Selected & 0.002441984 & UP \\
\hline$F Y N$ & FYN oncogene related to SRC, FGR, and YES & ENSP00000357656 & Connector & 0.005814608 & UP \\
\hline$T J P 1$ & Tight junction protein 1 (zona occludens 1) & ENSP00000281537 & Connector & 0.00617121 & $\mathbf{U P}$ \\
\hline$Q K I$ & Quaking homolog, KH domain RNA binding (mouse) & ENSP00000354951 & Selected & 0.010378886 & UP \\
\hline$O L I G 2$ & Oligodendrocyte transcription factor 2 & ENSP00000331040 & Selected & 0.011965689 & $\mathbf{U P}$ \\
\hline $\begin{array}{c}G J C 1 \\
(C X 45)\end{array}$ & Gap junction protein, gamma $1,45 \mathrm{kDa}(\mathrm{Cx} 45)$ & ENSP00000333193 & Selected & 0.020455465 & UP \\
\hline GLA & Galactosidase, $\alpha$ & ENSP00000218516 & Connector & 0.031337406 & DOWN \\
\hline$E P 300$ & microRNA 1281 & ENSP00000263253 & Connector & 0.041373019 & UP \\
\hline ASAH2 & $\mathrm{N}$-acylsphingosine amidohydrolase (non-lysosomal ceramidase) 2 & ENSP00000378897 & Connector & --- & --- \\
\hline CNP & 2',3'-cyclic nucleotide 3' phosphodiesterase & ENSP00000377470 & Selected & --- & --- \\
\hline$C R K$ & v-crk sarcoma virus CT10 oncogene homolog (avian) & ENSP00000300574 & Connector & --- & --- \\
\hline
\end{tabular}




\begin{tabular}{|c|c|c|c|c|c|}
\hline$G A L C$ & Galactocerebroside C & ENSP00000261304 & Selected & --- & --- \\
\hline ITGAV & Integrin, $\alpha \mathrm{V}$ (vitronectin receptor, $\alpha$ polypeptide, antigen CD51) & ENSP00000261023 & Connector & --- & --- \\
\hline OTUD4 & OTU domain containing 4 & ENSP00000409279 & Connector & --- & --- \\
\hline$P R K C D$ & Protein kinase $\mathrm{C}, \delta$ & ENSP00000331602 & Connector & --- & --- \\
\hline$P R N P$ & Prion protein & ENSP00000368748 & Connector & --- & --- \\
\hline SMAD2 & SMAD family member 2 & ENSP00000262160 & Connector & --- & --- \\
\hline WASF1 & WAS protein family, member 1 & ENSP00000352425 & Selected & --- & --- \\
\hline
\end{tabular}


A

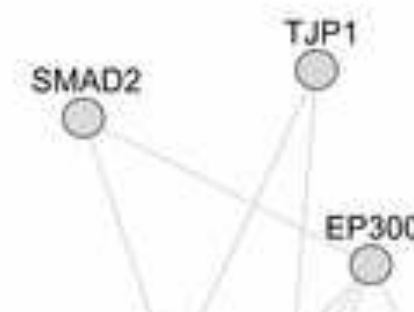

Number of nodes: 30

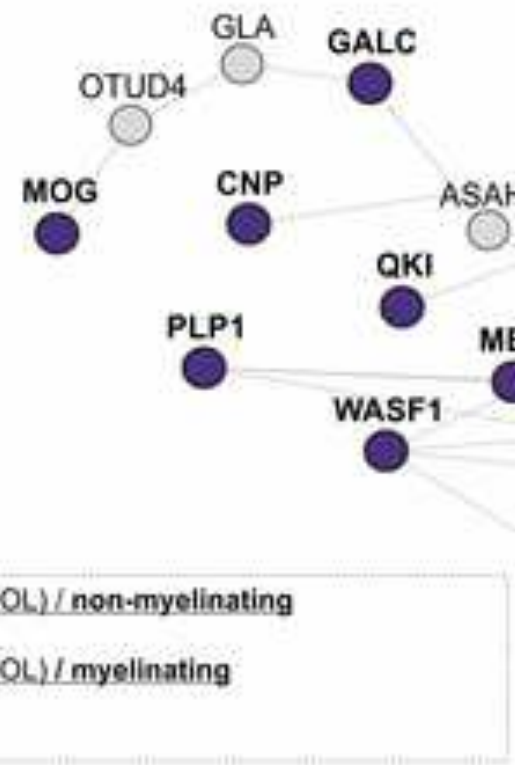

- Oligodendrocste (OL) / non-myelinating

- Olgodendrocyte(OL)/ myelinating

- Connectors

CRK

PDGFRA
B

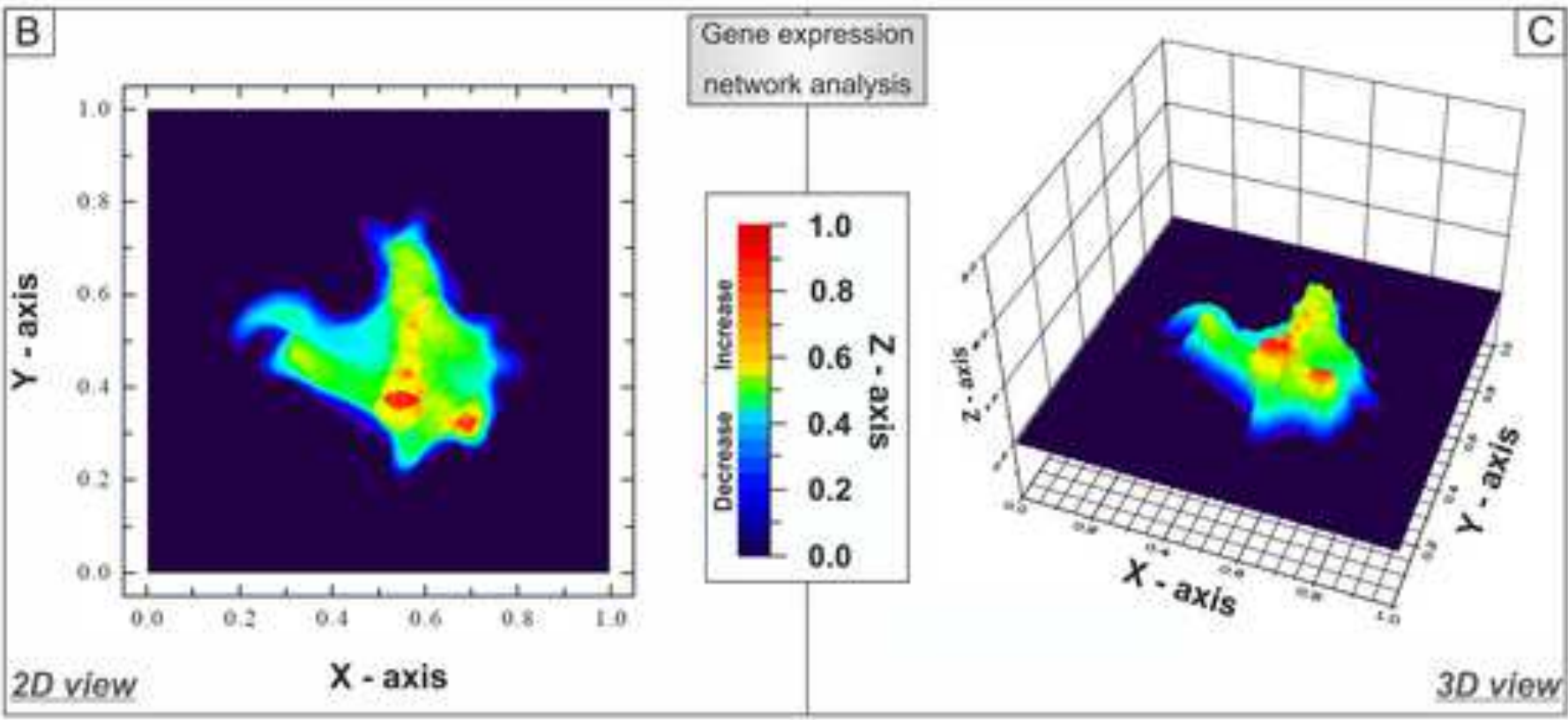

PRKCD

PRMT1

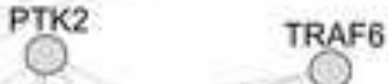

FYN

SPHK1

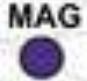

CSPG4

O

OLIG2

$\stackrel{\text { GJC1 }}{\text { OLIG1 }}$

ITGAV

$\mathrm{COC}^{\mathrm{C} 2}$

GRB2

PRNP

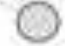

O 
Click here to download Figure: Figure 2.tif

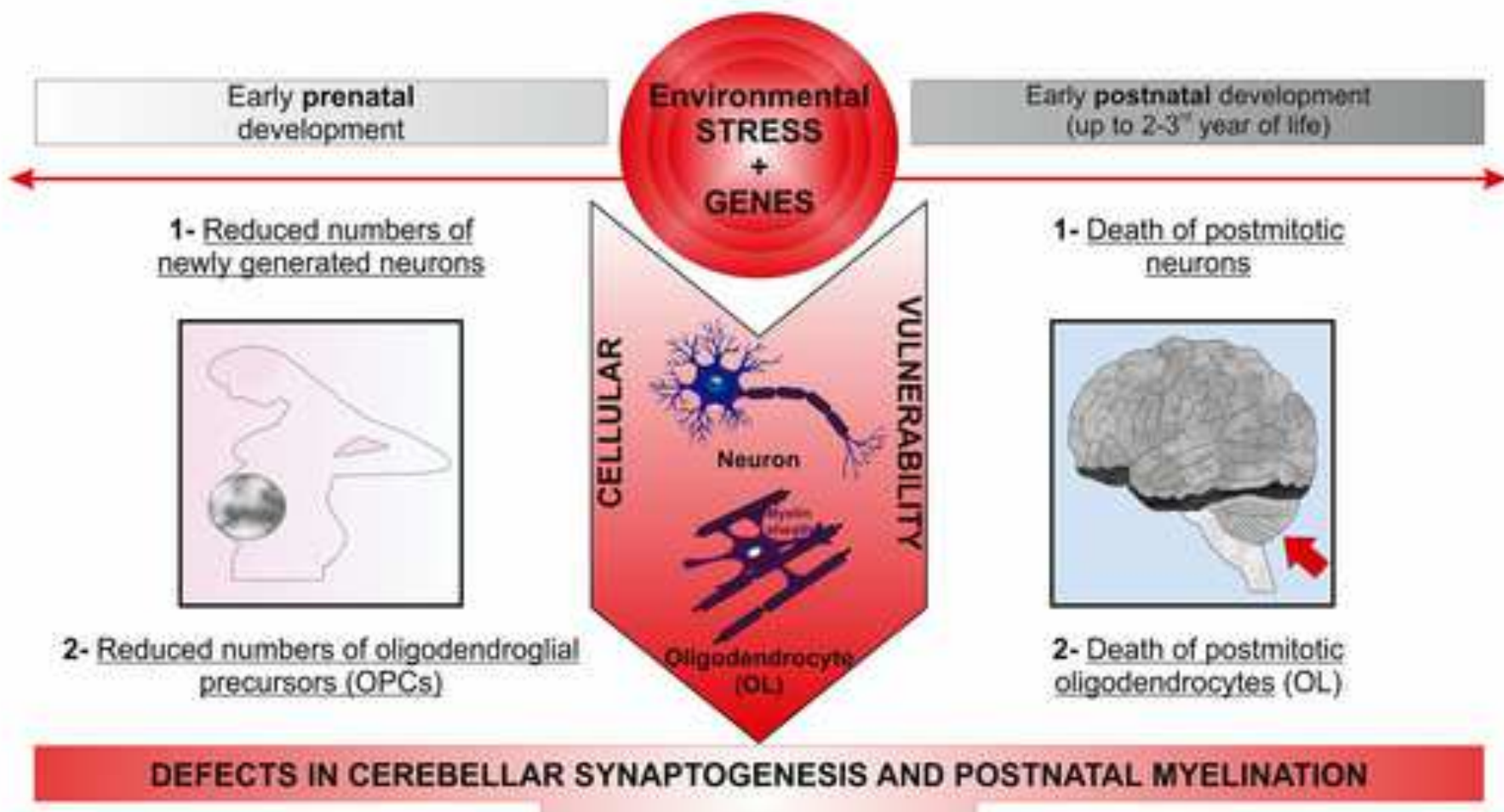

\section{Adaptive response to stress-induced cell death}

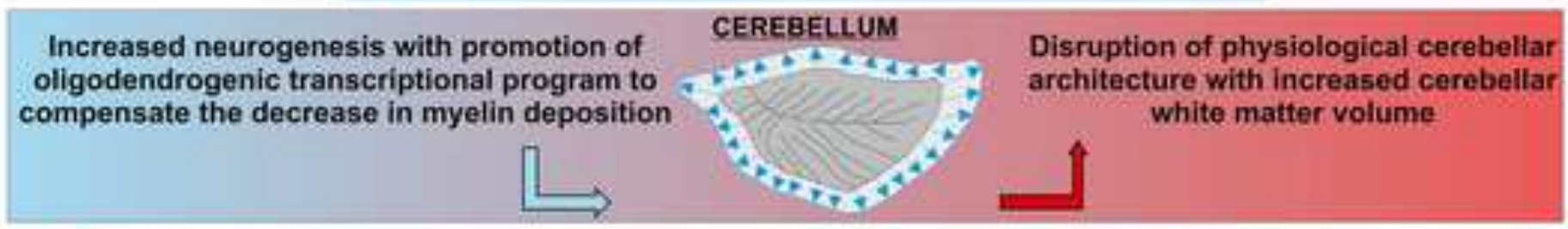

\section{DISRUPTION OF SYNAPTIC PLASTICITY AND CONNECTOME}
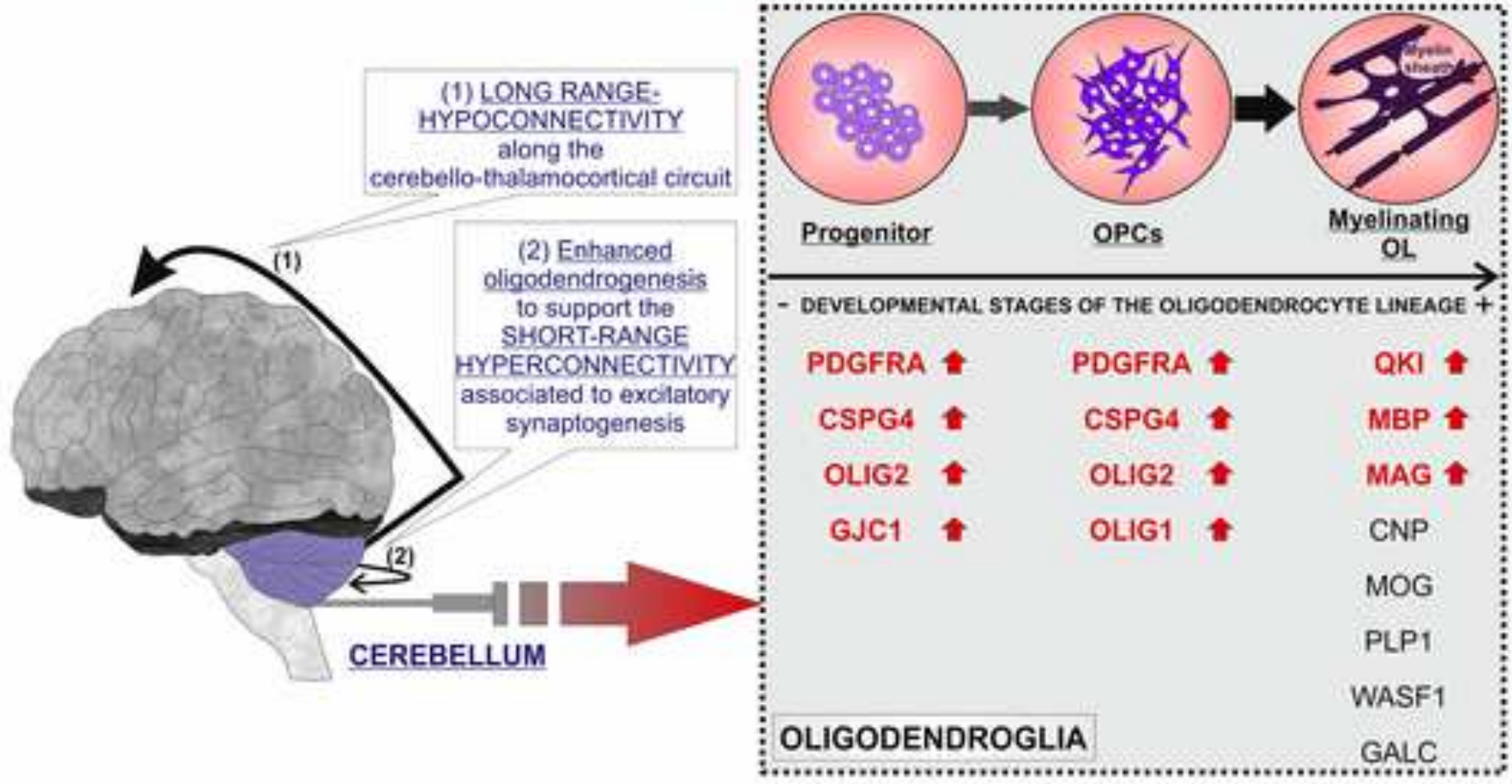